\title{
The Burden of Pneumococcal Pneumonia - Experience of the German Competence Network CAPNETZ
}

\author{
Epidemiologie, Klinik und Verlauf der Pneumokokkenpneumonie - \\ Daten aus CAPNETZ
}

Authors

Institutions
M. W. Pletz ${ }^{1, *}$, H. von Baum ${ }^{3, *}$, M. van der Linden ${ }^{4, *}$, G. Rohde ${ }^{5, *}$, H. Schütte ${ }^{6,{ }^{*}}$, N. Suttorp ${ }^{6, *}$, T. Welte ${ }^{2, *}$

Institutions are listed at the end of article. received 15.6. 2012 accepted after revision 27. 6. 2012

\section{Bibliography}

Dol http://dx.doi.org/

$10.1055 / \mathrm{s}-0032-1310103$

Pneumologie 2012; 66: 470-475

(c) Georg Thieme Verlag KG Stuttgart · New York

ISSN 0934-8387

Corresponding author Prof. Dr. Mathias W. Pletz

Universitätszentrum für Infektionsmedizin und Krankenhaushygiene

Universitätsklinikum Jena

Erlanger Allee 101

07740 Jena

Germany

mathias.pletz@med.uni-jena.de

\section{Abstract \\ $\nabla$}

Background: Pneumococcal pneumonia is still an important cause of mortality. The objective of this study was to compare frequency, clinical presentation, outcome and vaccination status of patients with pneumococcal community-acquired pneumonia (CAP) to CAP due to other or no detected pathogen based on data of the German Network for community-acquired pneumonia (CAPNETZ). Methods: Demographic, clinical and diagnostic data were recorded using standardized webbased data acquisition. Standardized microbiological sampling and work-up were conducted in each patient.

Results: 7400 patients with CAP from twelve clinical centers throughout Germany were included. In 2259 patients (32\%) a pathogen was identified, Streptococcus pneumonia being the most frequent ( $n=676,30 \%$ of all patients with identified pathogens). Compared to those with non-pneumococcal pneumonia, patients with pneumococcal pneumonia were more frequently admitted to hospital ( $80 \%$ vs. $66 \%, \mathrm{p}<0.001)$, had higher CURB score values on admission, had more frequently pleural effusion ( $19 \%$ vs. $14 \%, p=0.001$ ) and needed more frequently oxygen insufflation ( $58 \%$ vs. $44 \%, \mathrm{p}<0.001$ ). There was no relevant difference in overall mortality.

Conclusions: Pneumococcal pneumonia was associated with a more severe clinical course demanding more medical resources as compared to nonpneumococcal pneumonia.

\section{Introduction}

$\nabla$

Despite antibiotic treatment, community-acquired pneumonia remains a disease with considerable morbidity and mortality [1]. A study of the German competence network on community-ac-

\section{Zusammenfassung}

$\nabla$

Hintergrund: Trotz Verfügbarkeit wirksamer Antibiotika ist die Pneumokokkenpneumonie weiterhin mit einer relevanten Mortalität belastet. In der vorliegenden Arbeit wurden Häufigkeit, Symptomatik, Verlauf und Impfstatus von Patienten mit ambulant erworbener Pneumonie mit und ohne Nachweis von Pneumokokken verglichen.

Methoden: Demographische, klinische und diagnostische Daten wurden über ein internet-basiertes Formular erfasst. Bei jedem Patienten wurde eine standardisierte mikrobiologische Diagnostik durchgeführt.

Ergebnisse: 7400 Patienten mit ambulant erworbener Pneumonie wurden in 12 deutschen Studienzentren eingeschlossen. Bei 2259 Patienten $(32 \%)$ wurde ein Erreger identifiziert, dabei wurden Pneumokokken am häufigsten nachgewiesen ( $n=676,30 \%$ aller Patienten mit Erregernachweis). Patienten mit Pneumokokkennachweis wurden häufiger hospitalisiert ( $80 \%$ vs $66 \%$, $\mathrm{p}<0.001$ ), hatten einen höheren CURB-Punktwert bei Studieneinschluss, entwickelten häufiger einen Pleuraerguss ( $19 \%$ vs $14 \%, \mathrm{p}=0.001$ ) und benötigten häufiger eine Sauerstoffinsufflation ( $58 \%$ vs $44 \%$, p < 0.001 ). Es gab keinen signifikanten Unterschied in der Letalität.

Schlussfolgerung: Patienten mit Pneumokokkenpneumonien zeigten einen schwereren Verlauf und erforderten eine komplexere medizinische Versorgung als Patienten, bei denen keine Pneumokokken nachgewiesen wurden.

quired pneumonia (CAP), CAPNETZ, revealed that $13.8 \%$ of patients hospitalized with CAP die [2]. For decades, most studies investigating the etiology of CAP have identified Streptococcus pneumoniae as the most frequent pathogen in CAP [3].

\footnotetext{
* for the CAPNETZ Study Group
} 
Most recent studies confirmed the impact of this pathogen and revealed that molecular methods are of increasing importance in the diagnosis of pneumococcal pneumonia [4]. Besides influenza, S. pneumoniae is currently the only pathogen in community-acquired pneumonia that can be targeted by a licensed vaccine [5].

Since the mortality of pneumococcal pneumonia has not been changing for many years despite available antimicrobial agents with proven in vitro activity [6-8], prevention of pneumococcal infection by vaccination seems to be a rational approach in order to further decrease the public burden of disease.

This analysis aims to investigate the characteristics, course and outcome of patients with pneumococcal pneumonia who were enrolled into the CAPNETZ cohort between 2002 and 2008 and compares them to patients with non-pneumococcal pneumonia. To describe the burden of pneumococcal pneumonia, patients were divided into two groups: patients with pneumococcal pneumonia (CAP-P) and patients in whom other, non-pneumococcal pathogens or no pathogens were detected (CAP-nP).

\section{Patients and Methods}

$\nabla$

Patient population

The inclusion criteria for the CAPNETZ study were age $\geq 18$ years, the presence of a new infiltrate on chest radiography, and at least 1 of the following criteria: history of fever (temperature $\geq 38.3^{\circ} \mathrm{C}$ ), cough, production of purulent sputum, or focal chest signs on auscultation. Patients who had been hospitalized during the 28 days preceding the study because of severe immunosuppression or active tuberculosis were excluded. The study was approved by the ethical review board of each participating clinical center, and all patients included gave informed consent.

\section{Data collection}

In this prospective study, all demographic, clinical and diagnostic data of the patients were recorded using standardized web-based data acquisition. The study period comprised 79 months starting on 1st June 2002 and ending 31st December 2008.

Pneumococcal vaccination status was considered positive when patients had received pneumococcal vaccine within the last 5 years prior to enrollment.

\section{Processing of samples}

All available respiratory specimens and blood cultures were immediately processed in the local microbiology laboratories of the participating clinical centers. Gram staining and culture were performed for all respiratory samples. Validated sputum, blood culture samples, pleural fluid, and undiluted and serially diluted tracheobronchial aspirates, protected-specimen brush (PBS), and broncho-alveolar lavage fluid (BAL) samples were plated on blood-sheep agar, CDC anaerobic blood agar and chocolate agar. Undiluted PBS and BAL fluid samples were also cultured on charcoal-yeast extract agar if Legionella spp. was suspected. All Gramnegative pathogens were identified to species level according to standard methods. Urine was tested for the presence of S. pneumoniae and Legionella spp. antigens using the Binax Now test and Legionella Now test (Binax Inc), respectively. Standardized throat washings of all patients using sterile $0.9 \% \mathrm{NaCl}$ were sent immediately to the German reference center for influenza in Berlin.

\section{RNA extraction and complementary DNA (cDNA)} synthesis

Viral RNA was extracted using a commercial kit (QIAamp Viral RNA Kit, Qiagen, Hilden, Germany). Briefly: $150 \mu \mathrm{L}$ of clinical specimen (throat swab, nasal swab or gargle) were mixed with an equal volume of lysis buffer $\mathrm{AL}$, heated for $15 \mathrm{~min}$ at $70^{\circ} \mathrm{C}$ and applied to a spin column. Unbound material was removed by several washing steps, and the RNA eluted using $50 \mu \mathrm{L}$ of RNAse-free water. The cDNA synthesis was carried out at $37^{\circ} \mathrm{C}$ for 1 hour using $10 \mu \mathrm{L}$ of RNA, $100 \mathrm{U}$ of murine leukemia virus reverse transcriptase \{Gibco BRL, Life Technologies GmbH, Karlsruhe, Germany), $10 \mathrm{mM}$ dithiothreitol, $150 \mu \mathrm{M}$ (each) dATP, dCTP, dGTP, and dTTP [20 U RNAsin (Promega, Germany)] and $0.25 \mu \mathrm{M}$ random hexamer primers\}.

\section{PCR and sequence analysis}

The TaqMan-PCR was carried out in a 96-well flat-bottomed microtiter plate format (Perkin-Elmer). The PCR mix was made up to a volume of $25 \mu \mathrm{L}$, containing $5 \mu \mathrm{L}$ of cDNA, $50 \mathrm{mM}$ Tris-hydrochloride, $\mathrm{pH}$ 9, $50 \mathrm{mM} \mathrm{KCl}, 4 \mathrm{mM} \mathrm{MgCl}_{2}, 0.2 \mathrm{mM}$ (each) dATP, dCTP, dGTP dUTP, 0.5 units uracil- $N$-glycosylase (UNG) (Gibco BRL, Life Technologies, Germany), 1.25 units Taq DNA polymerase (InViTek, Berlin, Germany), $0.25 \mu \mathrm{M}$ each of the forward and reverse primer, $0.2 \mu \mathrm{M}$ of a fluorescence-labelled probe and $1 \mu \mathrm{M}$ ROX as passive reference. Virus identification and further subtyping was carried out as described previously with some modifications (primer and probe sequences on request) [9]. The cDNA was amplified by 45 two-step cycles $\left(1 \mathrm{~min} 92^{\circ} \mathrm{C}, 1 \mathrm{~min} 60^{\circ} \mathrm{C}\right)$. The amplification in the TaqMan-PCR was followed on the $\mathrm{ABI}$ PrismTM 7700 Sequence Detector (Applied Biosystems, Foster City, Calif. USA). The plate was scanned at $518 \mathrm{~nm}$ (FAM) and $582 \mathrm{~nm}$ (TAMRA). Data acquisition analysis was handled by using the Fluorescence Data Manager (Perkin-Elmer) and Excel (Microsoft Corporation, Redmond, WA) spreadsheets. ROX was used as a passive reference to which the reporter dye signal was normalized (Rn) during data analysis.

\section{Definitions}

S. pneumoniae was considered as pathogen when (i) isolated from blood cultures or pleural fluid cultures or (ii) in the presence of a good quality sputum revealing $>25$ polymorphonuclear cells and $<10$ epithelial cells per power field (total magnification $\times 100$ ) and predominant growth in culture of sputum $\left(\geq 10^{6} \mathrm{cfu}\right.$ ) or BAL $\left(\geq 10^{4} \mathrm{cfu} / \mathrm{mL}\right.$ ) or (iii) when the antigen was detected in urine.

Analyses were based on the fact whether S. pneumoniae was detected in any microbiological assay or not: "S. pneumoniae detected" (CAP-P) or "non-S. pneumoniae detected" (CAP-nP).

\section{Statistical analysis}

Comparisons between groups were performed by means of the chi square test for categorical variables or Fisher's exact test in case of small expected frequencies and analysis of variances (ANOVA) for continuous variables including multiple comparisons. Multivariate analysis of predictive factors for 30-day mortality and CAP due to $S$. pneumoniae was performed using binary logistic regression analysis. All analyses were performed with SPSS software (SPSS 10.0, Chicago, IL). All tests of significance were 2tailed, and alpha was set at 0.05 . 
Table 1 Demographics and risk factors.

\begin{tabular}{|llll|}
\hline & CAP-P & CAP-nP & Statistics \\
\hline Mean age [years $\pm \mathrm{SD}]$ & $59.8 \pm 17.8$ & $59.7 \pm 18.4$ & n.s. \\
\hline Mean BMI $\left[\mathrm{kg} / \mathrm{m}^{2} \pm \mathrm{SD}\right]$ & $24.4 \pm 4.6$ & $25.7 \pm 5.3$ & n.s. \\
\hline Males & $55.5 \%$ & $55.8 \%$ & n.s. \\
\hline Smokers & $40.2 \%$ & $30.0 \%$ & $\mathrm{p}<0.001$ \\
\hline Nursing home residents & $7.4 \%$ & $7.2 \%$ & n.s. \\
\hline Cadiac co-morbidities & $16.7 \%$ & $18.6 \%$ & n.s. \\
\hline Diabetes mellitus & $18.0 \%$ & $16.0 \%$ & n.s. \\
\hline Renal co-morbidities & $7.6 \%$ & $8.2 \%$ & n.s. \\
\hline Hepatic co-morbidities & $5.9 \%$ & $3.1 \%$ & p $<0.001$ \\
\hline Respiratory co-morbidities & $39.4 \%$ & $35.4 \%$ & p $<0.05$ \\
\hline Cerebral co-morbidities & $9.8 \%$ & $11.1 \%$ & n.s. \\
\hline
\end{tabular}

\section{Results}

$\nabla$

Demographic characteristics, co-morbidities, risk factors and pneumococcal polysaccharide vaccination

Overall, 7400 patients with community-acquired pneumonia from twelve clinical centers throughout Germany were included in our analysis from 2002 to 2008. The 4108 male and $3202 \mathrm{fe}-$ male patients had a mean age of $60 \pm 18$ years. Sixty-nine percent of the patients were hospitalized when first contacted for participation in CAPNETZ. Eight percent of the patients were nursing home residents. Severity scores as assessed by CURB were available for $88.5 \%$ of the patients and distributed as follows: CURB 0 (35\%), $1(35 \%), 2(15 \%), 3(4 \%)$ and $4(0.5 \%)$, respectively (missing data $9.5 \%$. Two hundred and sixty-three patients (3.6\%) required mechanical ventilation.

Altogether, 238 patients (4.7\%) died within 30 days after diagnosis. 180 -day mortality was $9.5 \%$ (in $6.7 \%$ of the patients these data were not available).

Demographic characteristics and co-morbidities are displayed in - Table 1. CAP-P patients were significantly more often smokers and suffered more often from hepatic and respiratory co-morbidities.

\section{General microbial patterns}

In 387 patients data on microbiological testing were not available; these patients were excluded from further analysis.

In 2259 of the remaining 7013 patients ( $32.2 \%$ of all patients) a definite pathogen causing CAP could be identified.

S. pneumoniae was confirmed as the predominant respiratory pathogen in the study population (29.9\% of all patients with a causative pathogen identified): it was detected as single pathogen in 529 patients and in additional 147 patients with polymicrobial infections. Of the 147 patients with polymicrobial infections, S. pneumoniae was classified as the leading pathogen in 106 cases.

In the "CAP-nP" group other pathogens were detected in 1583 patients with Mycoplasma pneumoniae as the most frequent pathogen (46\%) followed by Legionella pneumophila (17\%), influenza viruses (10\%) and Haemophilus influenzae (7\%). All other pathogens accounted for less than $5 \%$, respectively, of all CAP with identified pathogens. In 4754 patients all microbiological analyses remained negative.
Microbiological detection of S. pneumoniae infections In 434 of 676 (64\%) patients with pneumococcal pneumonia, the pneumococcal antigen was detected in urine. In 182 patients $(27 \%)$ pneumococci were detected in sputum, and in 85 patients (13\%) there was a positive blood culture.

Signs, symptoms, chest-X-ray, laboratory values and CURB classification on admission

CAP-P patients presented more frequently with confusion, dyspnoea, fever and thoracic pain, and had more often purulent sputum. The proportion of CAP-P patients in CURB classes 2, 3 and 4 was higher and for CURB class 0 lower than for CAP-nP patients $(\bullet$ Fig. 1). Chest X-rays at the day of enrollment revealed more frequently parapneumonic effusion in CAP-P patients. CRP, BUN, WBC, serum glucose were significantly increased whereas serum sodium level was decreased in CAP-P patients ( $\bullet$ Table 2 ).

\section{Clinical course and outcome}

Significantly more patients with pneumococcal pneumonia required hospitalization, mechanically ventilation and oxygen insufflation ( $\bullet$ Table 3 ).

There was no significant difference regarding mortality between the groups. Chi square testing for CAP-P versus CAP-nP and death within $7,14,30$ and 180 days revealed no statistically significant difference ( $\bullet$ Table 4). However, there was a trend for increased mortality in the CAP-P group within the first 30 days. Accordingly, survival curves demonstrate an earlier sharper decrease in the CAP-P group ( $\bullet$ Fig. 2 ).

$75.9 \%$ of the non-survivors in the CAP-P group died within the hospital (5.6\% at home and $7.4 \%$ in nursing home) compared to $66.1 \%$ of non survivors in the CAP-nP group $(9.9 \%$ at home and $10.1 \%$ in nursing home).

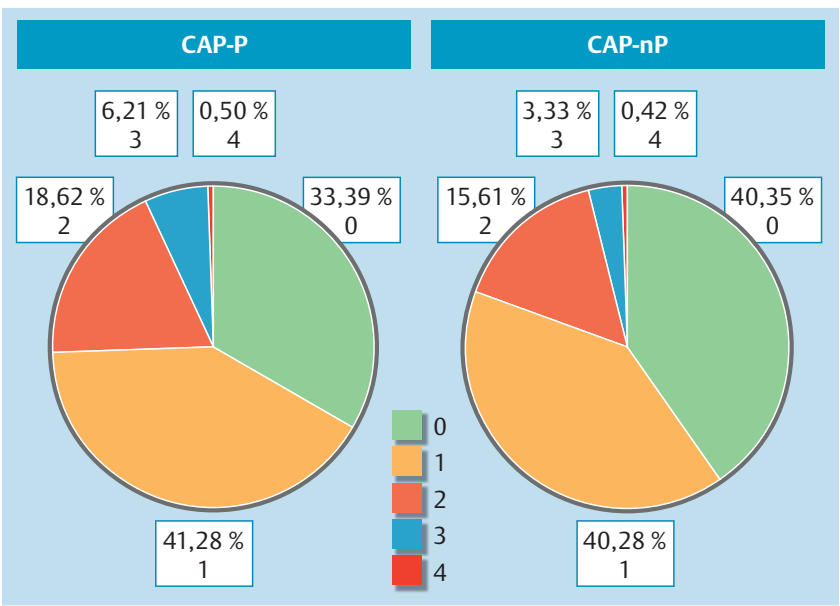

Fig. 1 CURB classification on admission.

Table 2 Signs, symptoms, chest-X-ray, laboratory values on admission.

\begin{tabular}{|llll|}
\hline & CAP-P & CAP-nP & Statistics \\
\hline Confusion & $13.4 \%$ & $9.2 \%$ & $\mathrm{p}=0.001$ \\
\hline Dyspnoea & $78.9 \%$ & $73.4 \%$ & $\mathrm{p}=0.002$ \\
\hline Purulent sputum & $66.8 \%$ & $55.5 \%$ & $\mathrm{p}<0.001$ \\
\hline Fever & $63.3 \%$ & $56.8 \%$ & $\mathrm{p}=0.001$ \\
\hline Pleural effusion on chest X-ray & $18.7 \%$ & $13.9 \%$ & $\mathrm{p}=0.001$ \\
\hline WBC $\left(10^{3} / \mu \mathrm{L}\right)$ & 15.3 & 12.2 & $\mathrm{p}<0.001$ \\
\hline C-reactive protein $(\mathrm{mg} / \mathrm{L})$ & 202 & 111 & $\mathrm{p}<0.001$ \\
\hline
\end{tabular}


Table 3 Clinical course.

\begin{tabular}{|lrrc|}
\hline Parameters & CAP-P & CAP-nP & Statistics \\
\hline Hospitalization & $79.7 \%$ & $66.2 \%$ & $\mathrm{p}<0.001$ \\
\hline Supplementary oxygen & $58.2 \%$ & $43.9 \%$ & $\mathrm{p}<0.001$ \\
\hline $\begin{array}{l}\text { Mechanical ventilation } \\
\text { (invasive and non-invasive) }\end{array}$ & $5.0 \%$ & $2.7 \%$ & $\mathrm{p}=0.001$ \\
\hline
\end{tabular}

Table 4 Outcome.

\begin{tabular}{|c|c|c|}
\hline & CAP-P & CAP-nP \\
\hline 30 day mortality & $4.9 \%$ & $4.0 \%$ \\
\hline 180 day mortality & $7.5 \%$ & $8.2 \%$ \\
\hline Died at hospital & $75.9 \%$ & $66.3 \%$ \\
\hline Died at nursing home & $7.4 \%$ & $10.1 \%$ \\
\hline Died at home & $5.6 \%$ & $9.9 \%$ \\
\hline
\end{tabular}

All comparisons were not significantly different.

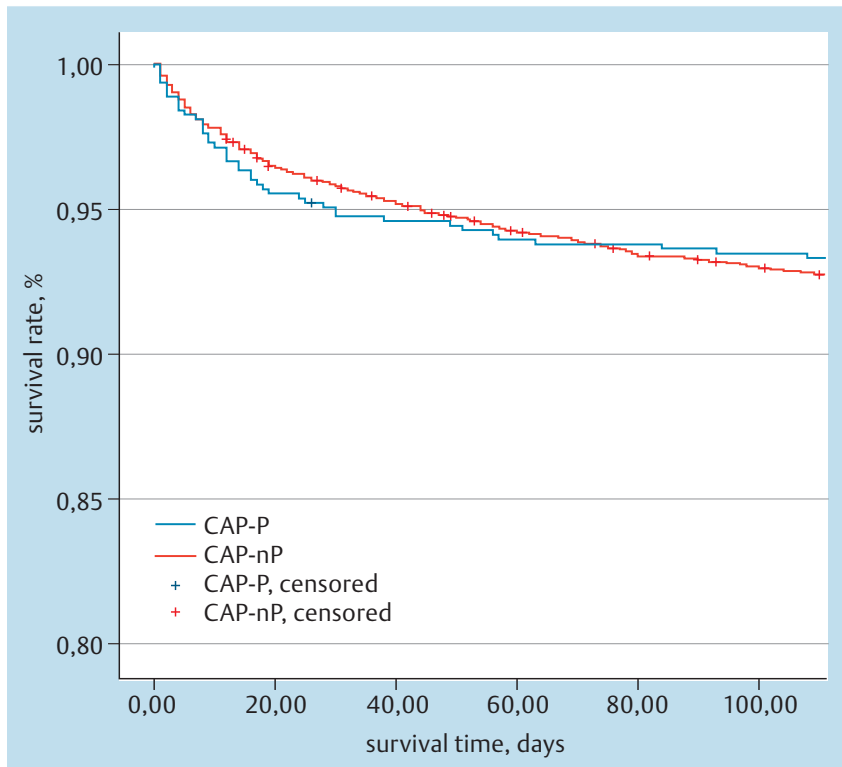

Fig. 2 Survival rate (Kaplan-Meier) of patients with CAP-P and CAP-nP.

\section{Vaccination status}

There were no significant differences regarding vaccination: $31.6 \%$ of the CAP-P and $35.4 \%$ of the CAP-nP patients had been vaccinated against influenza within the past 12 months. Pneumococcal polysaccharide vaccination within the past 5 years was received by $11.4 \%$ of the CAP-P patients and $12.1 \%$ of the CAP-nP patients.

There was no significant difference regarding pneumococcal polysaccharide vaccination between CAP-P and CAP-nP, survivors and non-survivors, and out- and inpatients, respectively ( Table 5). A subanalysis of patients older than 60 years - in Germany influenza and pneumococcal polysaccharide vaccination are recommended for all people at the age of 60 and above - also revealed no relevant advantage of the pneumococcal polysaccharide vaccination for patients with CAP-P: 5 of 53 vaccinated patients died (8.6\%) versus 23 of 235 unvaccinated patients (8.9\%). In contrast, vaccinated patients had a significantly decreased rate of pneumococcal bacteraemia (OR 0.28, $95 \%$ CI 0.09 to 0.90$)(\bullet$ Table 6$)$.
Table 5 Impact of vaccination by PPV-23 on hospitalization and outcome. Patients with missing data on vaccination status, hospitalization status or outcome were excluded.

\begin{tabular}{|lllll|}
\hline & $\begin{array}{l}\text { CAP-P } \\
\text { survived } \\
\text { (n, \%) }\end{array}$ & $\begin{array}{l}\text { died } \\
\text { (n, \%) }\end{array}$ & $\begin{array}{l}\text { CAP-nP } \\
\text { survived } \\
\mathbf{( n , \% )}\end{array}$ & $\begin{array}{l}\text { died } \\
\text { (n, \%) }\end{array}$ \\
\hline $\begin{array}{l}\text { Not } \\
\text { vaccinated }\end{array}$ & $480(95.0 \%)$ & $25(5.0 \%)$ & $4539(92.2 \%)$ & $383(7.8 \%)$ \\
\hline Vaccinated & $61(91.0 \%)$ & $6(9.0 \%)$ & $661(93.5 \%)$ & $46(6.5 \%)$ \\
\hline & $\begin{array}{l}\text { Outpatient } \\
\text { (n, \%) }\end{array}$ & $\begin{array}{l}\text { Inpatient } \\
\text { (n, \%) }\end{array}$ & $\begin{array}{l}\text { Outpatient } \\
\text { (n, \%) }\end{array}$ & $\begin{array}{l}\text { Inpatient } \\
\mathbf{( n , \% )}\end{array}$ \\
\hline $\begin{array}{l}\text { Not } \\
\text { vaccinated }\end{array}$ & $117(21.8 \%)$ & $419(78.2 \%)$ & $1840(36.0 \%)$ & $3273(64.0 \%)$ \\
\hline Vaccinated & $19(27.5 \%)$ & $50(72.5 \%)$ & $267(36.8 \%)$ & $459(63.2 \%)$ \\
\hline
\end{tabular}

All comparisons were not significantly different.

Table 6 Impact of vaccination by PPV-23 on rate of pneumococcal bacteraemia (Chi-square, $\mathrm{p}=0.022)$, OR $=0.28(95 \% \mathrm{Cl} 0.09-0.9) ; 728$ patients $(9.8 \%)$ with missing data on vaccination status were excluded.

\begin{tabular}{|c|c|c|c|}
\hline \multirow[t]{2}{*}{ PPV-23 status } & \multicolumn{3}{|c|}{ Pneumococcal bacteraemia? } \\
\hline & no & yes & \\
\hline Not vaccinated & $5774(98.7 \%)$ & $75(1.3 \%)$ & $5849(100 \%)$ \\
\hline Vaccinated & $820(99.6 \%)$ & $3(0.4 \%)$ & $823(100 \%)$ \\
\hline
\end{tabular}

Eighty-five percent of patients who had received the pneumococcal polysaccharide vaccination were also vaccinated against influenza whereas only $66 \%$ of patients who had not received the pneumococcal polysaccharide vaccine were vaccinated against influenza (chi square $\mathrm{p}<0.001$ ).

\section{Discussion}

In patients with moderate to severe CAP, morbidity and mortality remain a global problem: short-term mortality reaches $14 \%$ (7\% if nursing-home residents and bedridden patients are excluded), and long-term mortality $50 \%$ within five years [10]. While past studies revealed that prompt initiation of expanded-spectrum antimicrobial therapy is essential for the prevention of unnecessary mortality and complications in patients, particularly in the elderly and other at-risk populations [11], new preventive strategies are needed to accomplish a reduction in CAP incidence and to reduce morbidity and mortality. To analyse the burden of pneumococcal pneumonia in adults and to investigate the impact of the available pneumococcal vaccine in the past decade, characteristics, course and outcome of patients with pneumococcal pneumonia were studied.

This analysis is based on data of the German Network for Community Acquired Pneumonia (CAPNETZ), one of the largest prospective surveillance studies for the management of inpatients and outpatients with CAP worldwide. In contrast to other large surveillance studies, only patients with radiologically confirmed pneumonia are included. In fact, our data are in line with other studies showing that $S$. pneumoniae remains the most frequent pathogen in CAP, regardless of concerned patient group [12] (for review see [3]). Despite this, we did not detect a higher overall mortality in pneumococcal pneumonia patients compared to those with other or no detected pathogens. However, patients with pneumococcal pneumonia had a significant more severe 
course of disease, e.g., more frequently parapneumonic effusion, higher CURB score on admission - CURB was chosen instead of CRB-65 because we wanted to assess the severity of disease independently of age. Age is a predictor of mortality, but not a parameter of severity by itself, and more resources (hospitalization, mechanical ventilation, oxygen insufflation) were required to treat these patients. An analysis regarding length of stay for hospitalized patients was not performed because the length of hospital stay may be biased by economical factors of the German reimbursement system.

While PPV-23 vaccination protects - according to a Cochrane meta-analysis - against invasive pneumococcal diseases (OR $0.26,95 \%$ CI 0.15 to 0.46 ) such as bacteraemic pneumonia, data on non-bacteraemic pneumonia are inconclusive [13-16]. In elderly nursing-home residents in Japan (mean age $>84$ years), a randomized controlled trial recently demonstrated a benefit for PPV-23 in regard of the prevention of pneumococcal pneumonia [17].

Our data are in line with these findings, with PPV-23 vaccinated patients exhibiting a significantly reduced rate of pneumococcal bacteraemia with an OR similar to that of -analysis mentioned above (OR.28, 95\%CI 0.09 to 0.90 ). In our study, only a minority of patients with an indication for pneumococcal and influenza vaccination had actually received these vaccinations. This may explain the limited protective effect of PPV-23 in regard of mortality or hospitalization: Due to the low vaccination rate an underlying selection bias towards individuals with more co-morbidities cannot be excluded and could result in higher mortality in vaccinated patients. However, in both pneumococcal and nonpneumococcal pneumonia, vaccination rates were similar. Despite the possible selection bias, these findings are in line with several meta-analyses and a recently published cohort study demonstrating minor or no efficacy of PPV-23 in regard of noninvasive pneumococcal pneumonia [13-16].

Our analysis is limited by following issues: severity and outcome of disease are influenced by three factors: pathogen, patient and treatment. Since certain patient characteristics and risk factors predispose for certain pathogens (e.g., M. pneumoniae is more frequently diagnosed in younger patients) [18] it is difficult even with a large data base - to asses the contribution of an individual pathogen to outcome in comparison to all other causes of CAP. Another factor influencing our results is caused by different sensitivity and specificity of microbiological methods for certain pathogens. Particularly, the sensitivity of diagnostic procedures to detect S. pneumoniae is insufficient $[19,20]$. The reader should be aware that within the CAP-nP group there may be numerous patients with pneumococcal pneumonia that has not been detected despite blood culture, sputum culture and urine antigen test. However, due to the different characteristics of patients infected with certain pathogens (e.g., significantly younger age of patients infected with Mycoplasma spp.) we used our group stratification, which may present an approach more reflecting every day clinical practice.

In conclusion, S. pneumoniae was the most frequent cause of CAP in our study. Pneumococcal pneumonia was associated with a more severe course demanding more medical resources than non-pneumococcal pneumonia.

\section{Conflict of Interest}

$\nabla$

CAPNETZ is a multidisciplinary approach to better understand and treat patients with community-acquired pneumonia. The network is funded by the German Ministry of Education and Research (Bundesministerium für Bildung und Forschung), grant number 01KI07145. M. W. Pletz is supported by the German Ministry of Education and Research (Bundesministerium für Bildung und Forschung, BMBF, grant No.01 KI 1204). The study was supported by an unrestricted grant from Wyeth (Wyeth is now part of Pfizer). The network has only been made possible by the contribution of many investigators. Members of the CAPNETZ study group except the authors are: T. Bauer, F. Kunitz (HELIOS Klinikum Emil von Behring, Berlin); B. Hauptmeier, S. Ewig (Thoraxzentrum Ruhrgebiet, Department of Respiratory and Infectious Diseases, EVK Herne and Augusta-Kranken-Anstalt Bochum, Germany); C. Schumann (Department of Internal Medicine II, University of Ulm); T. Schaberg, I. Hering (Center of Pneumology, Diakonie-Hospital Rotenburg); K. Dalhoff, P. Heyer (Med. Clinic III, Pulmology, University Clinic Schleswig-Holstein, Lübeck); M. Prediger, K. Kaube (III. Medical Clinic, Carl-Thiem-Klinikum Cottbus); T. Welte, M. Pletz, J. Rademacher (Department of Respiratory Medicine, Hanover Medical School, Hanover); B. Drewelow (Center of Pharmacology and Toxicology, Institute of Clinical Pharmacology, University of Rostock); N. Suttorp, A. Tessmer (Department of Infectious Disease and Respiratory Medicine, Charité-University Medicine, Berlin); O. Burghuber, G. Rainer (Internal Lung Department, Otto Wagner Spital Wien); W. Petermann, H. Buschmann, R. Kröning, Y. Aydin (Brotherhospital St. Josef, Medical Clinic - Pneumology, Paderborn); S. Krüger (Medical Clinic I, University Clinic RWTH Aachen); W. Pankow, A. Lies (Clinic for Internal Medicine - Pneumology and Infektiology - Vivantes Klinikum Neukölln); R. Marre (University of Ulm), G. Rohde (Department of Respiratory Medicine, Maastricht Universitary Medical Centre - MUMC+, Maastricht), R. Bals (University Clinic Saarlandes, Internal Medicine V - Pneumology, Homburg/ Saar), N. Suttorp, H. Schütte (Department of Infectious Disease and Respiratory Medicine, Charité-University Medicine, Berlin); G. Barten, L. Gosman (Main Office, Hannover); H. von Baum (Ulm University Hospital, Med. Microbiology and Hygiene); P. Martus (Institute for Biostatistics and Clinical Epidemiology, Charité University Medicine Berlin); T. Illmann, M. Wallner (2mt Software, Ulm) and all study nurses. It is also our responsibility and pleasure to express our appreciation to all clinical physicians and physicians in private practice who saw and identified patients with community acquired pneumonia for their work dedicated to CAPNETZ.

\footnotetext{
Institutions

${ }^{1}$ Center for Infectious Diseases and Infection Control, Jena University Hospital, Jena, Germany

2 Department of Pulmonary Medicine, Hannover Medical School, Hannover, Germany

${ }^{3}$ Institute of Medical Microbiology and Hygiene, University Hospital of Ulm, Ulm, Germany

${ }^{4}$ National Reference Center for Streptococci, Institute of Medical Microbiology, University Hospital (RWTH), Aachen, Germany

Department of Respiratory Medicine, Maastricht University Medical Centre MUMC, Maastricht, The Netherlands

${ }^{6}$ Department of Respiratory Medicine and Infectious Diseases, Charité

University Medicine, Berlin, Germany
} 


\section{References}

1 Trotter CL, Stuart JM, George R et al. Increasing hospital admissions for pneumonia, England. Emerg Infect Dis 2008; 14: 727-733

2 Welte T, Kohnlein T. Global and local epidemiology of community-acquired pneumonia: the experience of the CAPNETZ Network. Semin Respir Crit Care Med 2009; 30: 127-135

3 Woodhead M. Community-acquired pneumonia in Europe: causative pathogens and resistance patterns. Eur Respir J Suppl 2002; 36: 20s $27 \mathrm{~s}$

4 Marchese A, Esposito S, Coppo E et al. Detection of Streptococcus pneumoniae and identification of pneumococcal serotypes by real-time polymerase chain reaction using blood samples from Italian children $\leq 5$ years of age with community-acquired pneumonia. Microb Drug Resist 2011; 17: 419-421

5 Pletz MW, Welte T, Ott SR. Advances in the prevention, management, and treatment of community-acquired pneumonia. F1000 Medicine Reports. [Review] 2010; 2: 53

6 Feikin DR, Schuchat A, Kolczak M et al. Mortality from invasive pneumococcal pneumonia in the era of antibiotic resistance, 1995-1997. Am J Public Health 2000; 90: 223-229

7 Austrian R, Gold J. Pneumococcal bacteremia with especial reference to bacteremic pneumococcal pneumonia. Ann Intern Med 1964; 60: $759-776$

8 Fine MJ, Smith MA, Carson CA et al. Prognosis and outcomes of patients with community-acquired pneumonia. A meta-analysis. JAMA 1996; 275: $134-141$

9 Schweiger B, Zadow I, Heckler R et al. Application of a fluorogenic PCR assay for typing and subtyping of influenza viruses in respiratory samples. J Clin Microbiol 2000; 38: 1552-1558

10 Ewig S, Torres A. Community-acquired pneumonia as an emergency: time for an aggressive intervention to lower mortality. Eur Respir J 2011; 38: $253-260$
11 Welte $T$. Risk factors and severity scores in hospitalized patients with community-acquired pneumonia: prediction of severity and mortality. Eur J Clin Microbiol Infect Dis

12 Polverino E, Dambrava P, Cilloniz C et al. Nursing home-acquired pneumonia: a 10 year single-centre experience. Thorax 2010; 65: 354-359

13 Johnstone J, Eurich DT, Minhas JK et al. Impact of the pneumococcal vaccine on long-term morbidity and mortality of adults at high risk for pneumonia. Clin Infect Dis 2010; 51: 15-22

14 Moberley SA, Holden J, Tatham DP et al. Vaccines for preventing pneumococcal infection in adults. Cochrane Database Syst Rev 2008: 01 CD000422

15 Johnstone J. ACP Journal Club. Review: pneumococcal vaccination is not effective for preventing pneumonia, bacteremia, bronchitis, or mortality.. Ann Intern Med 2009; 150: 5-14

16 Huss A, Scott P, Stuck AE et al. Efficacy of pneumococcal vaccination in adults: a meta-analysis. CMAJ 2009; 180: $48-58$

17 Maruyama T, Taguchi O, Niederman MS et al. Efficacy of 23-valent pneumococcal vaccine in preventing pneumonia and improving survival in nursing home residents: double blind, randomised and placebo controlled trial. BMJ 2010; 340: 1004

18 von Baum H, Welte T, Marre R et al. Mycoplasma pneumoniae pneumonia revisited within the German Competence Network for Community-acquired pneumonia (CAPNETZ). BMC Infect Dis 2009; 9: 62

19 Ruiz-Gonzalez A, Falguera M, Nogues A et al. Is Streptococcus pneumoniae the leading cause of pneumonia of unknown etiology? A microbiologic study of lung aspirates in consecutive patients with community-acquired pneumonia. Am J Med 1999; 106: 385 - 390

20 Ishida T, Hashimoto T, Arita $M$ et al. Etiology of community-acquired pneumonia in hospitalized patients: a 3-year prospective study in Japan. Chest 1998; 114: 1588-1593 